\title{
Billiard Model of a Ballistic Multiprobe Conductor
}

\author{
C. W. J. Beenakker and H. van Houten \\ Philips Research Laboratories, 5600 JA Eindhoven, The Netherlands
}

(Received 30 June 1989)

\begin{abstract}
A model for ballistic transport based on classical mechanics of electrons at the Fermi level is shown to exhibit a variety of magnetoresistance anomalies found experimentally in narrow-channel twodimensional electron gases. Among the phenomena considered are quenched and negative Hall resistances, the last Hall plateau, bend resistances, and geometrical resonances.
\end{abstract}

PACS numbers: $73.50 . \mathrm{Jt}, 72.20 . \mathrm{My}, 73.40 . \mathrm{Kp}$

Resistance measurements in a ballistic narrow channel in a two-dimensional electron gas show a complex, nonmonotonic dependence on a weak perpendicular magnetic field $B$. Phenomena which have drawn particular attention are the "quenching of the Hall effect"1-4 (a suppression of the Hall resistance around zero field), the "negative Hall resistance," 3 the "last Hall plateau" 1-4 (reminiscent of quantum Hall plateaus, but occurring at much lower B), "bend resistances" 5 (associated with current passing around the corner at a junction), and "magnetically reduced backscattering" (a decrease of the longitudinal resistance in weak magnetic fields). The theoretical effort in this field ${ }^{7-10}$ has focused on models of quantum-mechanical propagation and scattering, as in an electron waveguide. Quantum-mechanical phase coherence is certainly necessary for some of the fine structure which appears experimentally only at the lowest (mK) temperatures, but the phenomena listed above have a relatively weak temperature dependence - suggesting a different origin. In this Letter we demonstrate that a model based on classical junction scattering, as in an electron billiard, exhibits all these phenomena, which can thus be classified as classical magneto-size effects in a degenerate electron gas.

Our investigation builds on two recent papers: ${ }^{10,11}$ To explain the nonadditivity of the contact resistance of two opposite constrictions, we first pointed out ${ }^{11}$ that a flared (hornlike) constriction collimates the beam of injected electrons, as a result of the adiabatic invariance of the product of width and transverse momentum. Baranger and Stone have proposed ${ }^{10}$ (on the basis of a quantummechanical calculation of the low-field Hall resistance) that this collimation causes the quenching of the Hall effect in a (realistic) cross geometry with rounded corners, by suppressing the coupling of the currentcarrying channel to the side probes used to measure the Hall voltage.

We summarize our main results. Our calculations of the low-field Hall resistance $R_{H}$ show a quenched as well as a negative $R_{H}$, depending on the geometry and consistent with the experiments of Ford et al. ${ }^{3}$ in which different geometries were compared. We find that a strong suppression of the coupling to the side probes is not necessary for a drastic reduction of $R_{H}$ below its 2D value-a relatively weak collimation of the injected beam to a cone of $90^{\circ}$ angular opening being sufficient. At higher fields a strikingly broad and fiat Hall plateau appears-although the model contains no quantization. Its origin is the guiding-center drift along the curved channels walls at the junction. This classical effect enhances $R_{H}$ to the contact resistance of the lead, which is approximately independent of $B$ over a wide field range $^{12}$ - hence the plateau. Geometrical resonances cause oscillations on the Hall plateau, resembling the oscillations in the experiments. ${ }^{3,4}$ Magnetic guiding reduces backscattering, thereby suppressing the longitudinal resistance $R_{L}$ and the bend resistance $R_{B}$. As in the experiments ${ }^{13-15}$ we find an "overshoot" in $R_{B}$ from a negative to a positive value before it drops to zero, due to destruction of collimation before guiding becomes effective.

We consider the geometry of a long channel with two intersecting side channels (Fig. 1, right inset). An elec-

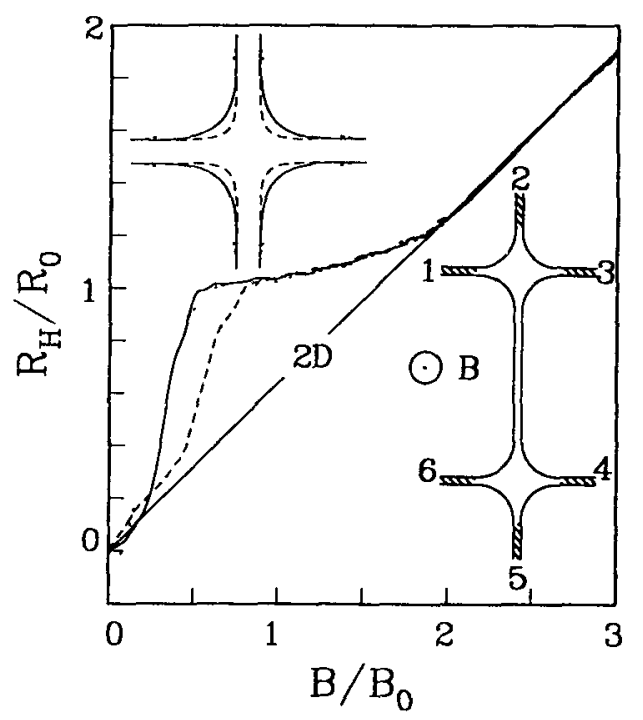

FIG. 1. Hall resistance for three hard-wall geometries. The straight line is the $2 \mathrm{D}$ result. The three curves are for a double-cross geometry (right inset), with different rounding of the corners (left inset; the contours are segments of the curve $x^{p}+y^{p}=$ const, with $p=2,4$, and 8 for the dotted, solid, and dashed contours, respectively). 
tron approaching a junction from one of the leads $i$ can leave through one of the other leads $j$, with transmission probability $T_{i \rightarrow j}$, or it can return through the same lead, with reflection probability $\boldsymbol{R}_{\boldsymbol{i}}$. If these probabilities are known (at the Fermi energy $E_{F}$ ), then the resistances follow from the Büttiker formula, ${ }^{16}$

$$
(h / 2 e) I_{i}=\left(N_{i}-R_{i}\right) \mu_{i}-\sum_{j \neq i} T_{J \rightarrow i} \mu_{J},
$$

which relates the current $I_{i}$ in lead $i$ to the chemical potential $\mu_{j}$ of the reservoir attached to lead $j$ (far from the junction). The transmission and reflection probabilities are normalized such that

$$
\sum_{j \neq i} T_{i \rightarrow j}+R_{i}=N_{i}
$$

with $N_{i}$ the number of propagating modes in lead $i$. In our case all leads are identical, so that $N_{i} \equiv N$ for all $i$. Note that in the semiclassical limit (including FermiDirac statistics but no quantization), $N$ is assumed large and continuous. Four-terminal resistances with source $i$, drain $j$, and voltage probes $k$ and $l$ are defined by $R_{i j, k l} \equiv\left(V_{k}-V_{l}\right) / I$, where $V_{k}-V_{l} \equiv\left(\mu_{k}-\mu_{l}\right) / e$ is obtained by solving Eq. (1) under the conditions that $I_{i}=-I_{j} \equiv I$, and $I_{m}=0$ for $m \neq i, j$.

To obtain the probabilities $T_{i \rightarrow j}$ and $R_{i}$ in the semiclassical limit, we simulate the injection of a large number (typically $10^{4}$ ) of electrons towards the junction through lead $i$, and follow their trajectories to determine the fractions $t_{i \rightarrow j}, r_{i}$ of electrons which leave via lead $j$, or return through the same lead $i$. The required probabilities then follow on normalization, $T-t N, R=r N$. The injection distribution is determined by the flux density injected into the lead by a reservoir in thermal equilibrium. In a hard-wall lead at $B=0$ the electrons are to be injected uniformly over the channel width $W$, with velocity magnitude $v_{F} \equiv\left(2 m E_{F}\right)^{1 / 2}$, and angular distribution $P(\alpha)=\frac{1}{2} \cos \alpha$, which is the angular distribution of flux in this case $[a$ in the interval $(-\pi / 2, \pi / 2)$ being the angle with the channel axis]. In this case $N$ equals $k_{F} W / \pi$ (with $k_{F} \equiv m v_{F} / \hbar$ the Fermi wave vector). For other confining potentials, or for $B \neq 0$, the injection distribution is different, and not easily calculated. We circumvent this difficulty by attaching to each channel of the structure a hard-wall lead in which $B=0$ (shaded in Fig. 1, right inset). This has no effect on the resistances in the semiclassical limit, ${ }^{17}$ while it permits us to use the simple injection distribution given above.

We show representative results calculated for three hard-wall geometries with various roundings of the corners (left inset in Fig. 1), and for a geometry defined by a smooth parabolic potential (inset in Fig. 2). The plots give resistances normalized by $R_{0} \equiv\left(h / 2 e^{2}\right) \pi / k_{F} W$ versus magnetic fields normalized by $B_{0} \equiv m v_{F} / e W$. (The width $W$ of the parabolic channel is defined as the separation of the equipotentials at $E_{F}$.) Note that $\rho \equiv R / R_{0}$ and $\beta \equiv B / B_{0}$ are the only two independent di-

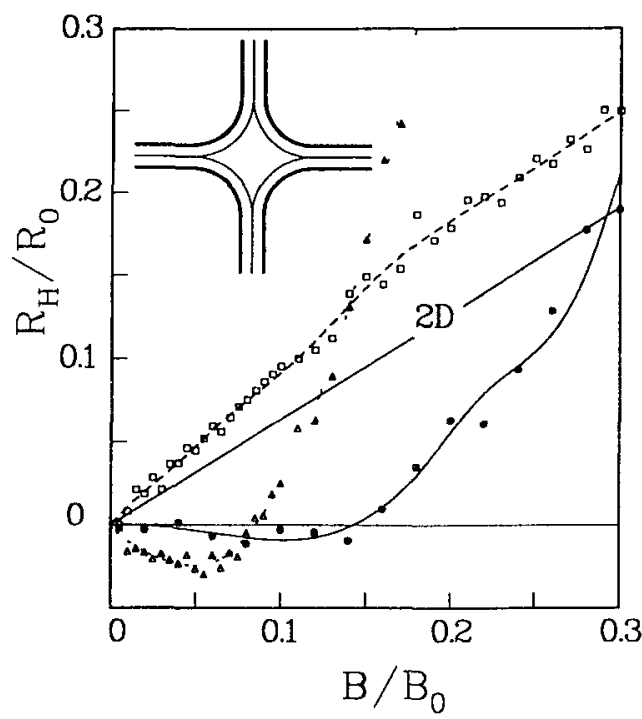

FIG. 2. Low-field Hall resistance for two hard-wall potentials (dashed and dotted curves, corresponding to Fig. 1), and for a parabolic potential [solid curve; inset: the equipotentials at $E_{F}$ (thick contour) and 0 (thin contour) - the potential vanishes in the diamond-shaped region at the center of the cross]. The individual data points give an indication of the numerical noise.

mensionless variables in our problem, so that a single trace fully represents our results for a given geometry (in a quantum theory, $k_{F} W$ appears as a third independent variable $\left.{ }^{9,10}\right)$. The results given are for $T=0$, but the temperature dependence is weak since phase coherence does not enter into the calculation. A finite temperature simply induces an average over the energy interval $\Delta E=3.5 k_{B} T$ (the width of the derivative of the Fermi function), which is approximately the average of $\rho$ over the interval $\Delta \beta=\frac{1}{2} \beta \Delta E / E_{F}$. The finest details in our magnetoresistance plots occur for $\beta \lesssim 1$ and require a resolution $\Delta \beta \gtrsim 0.1$, so that at temperatures $T-0.1 E_{F} /$ $k_{B} \sim 10 \mathrm{~K}$ these features are still resolved. This is in agreement with experiment. Note that the energy separation of the subbands does not enter in our criterion for the temperature dependence.

We first discuss the Hall resistance $R_{H} \equiv R_{25,31}$ at higher fields, shown in Fig. 1 for the three hard-wall geometries (the parabolic potential, not shown, gives similar results). For $B \gtrsim B_{\text {crit }} \equiv 2 B_{0}$ the data in Fig. 1 are on the straight line $R_{H} / R_{0}=(2 / \pi) B / B_{0}$, which is the classical $2 \mathrm{D}$ result. The field $B_{\text {crit }}$ is the field beyond which a cyclotron orbit with radius $l_{\text {cycl }}=m v_{F} / e B$ can no longer intersect both opposite channel walls. Experimental data are in general agreement with this classical characteristic field ${ }^{7}$ for the onset of deviations in $R_{H}$. At fields below $B_{\text {crit }}$, Fig. 1 shows a plateau of enhanced $R_{H}$. This is a prominent feature of experiments in narrow channels. ${ }^{1-4}$ Note that on the plateau $R_{H} \approx R_{0}$, independent of the rounding of the corners. The geometry 
does affect the width of the plateau, which persists down to lower $B$ for smoother corners. All these aspects agree with the recent experiments by Ford et al., ${ }^{3}$ in which narrow and widened crosses are compared. Our calculations show that the "last Hall plateau" has a classical origin, which is the guiding-center drift along the equipotentials of the confining potential in a sufficiently strong magnetic field $B>\boldsymbol{B}_{g}$ (see central inset in Fig. 3). We estimate $B_{g}=m v_{F} / e r_{\min }$, with $r_{\min }$ the minimal radius of curvature of the corners: When guiding is complete, the probability $T_{l}$ to turn left at the corner of a cross is maximal, $T_{l} \approx N$, while the probabilities to turn right, $T_{r}$, or move straight on, $T_{s}$, are both small, as noted also in Refs. 3 and 4. Equation (1) then tells us that $R_{H} \approx h / 2 e^{2} N \equiv R_{\text {contact }}$, where $R_{\text {contact }}$ is the contact resistance of the lead. Now the crux is that $R_{\text {contact }} \approx R_{0}$ is approximately independent of $B$ for $B \leqslant B_{\text {crt }}$ (see, e.g., Ref. 12), so that we obtain a classical Hall plateau at $R_{0}$, for $B_{g} \lesssim B \lesssim B_{\text {crtt. The oscillations on the plateau }}$ are due to geometrical resonances between $l_{\text {cycl }}$ and the radius of curvature of the corners (see Ref. 18 for a further identification). ${ }^{19}$ Similar oscillations occur experimentally. ${ }^{3,4}$

The behavior of $\boldsymbol{R}_{H}$ around zero field is qualitatively different depending on the geometry, as shown in Fig. 2 for the smoothest and least-smooth hard-wall geometries of Fig. 1, and for the smooth parabolic potential. In the geometry with relatively sharp corners, $R_{H}$ is enhanced over the 2D result. As discovered by Baranger and Stone ${ }^{10}$ smoothing the corners suppresses $R_{H}$. Both the quenched and the negative $R_{H}$ in Fig. 2 have been observed experimentally by Ford et al., ${ }^{3}$ in a narrow and

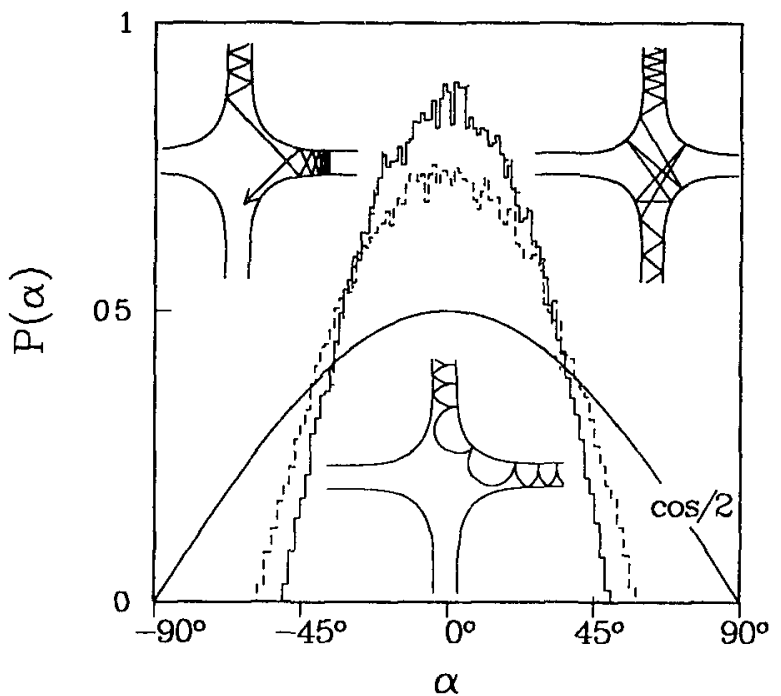

FIG. 3. Histograms of the angular distribution at $B=0$ of electrons injected into the junction, for the three hard-wall geometries of Fig.1. The cosine distribution for a rectangular junction (no collimation) is shown for comparison. The left, central, and right insets illustrate collimation, guiding, and scrambling of trajectories, respectively. widened cross, respectively. The effect of the smooth corners, for small $B<B_{g}$, is to collimate ${ }^{11}$ the electrons injected into the junction (see Fig. 3). We propose that the classical suppression of $R_{H}$ occurs for collimation to within an injection or acceptance cone of $\Delta \alpha=90^{\circ}$ angular opening, which is a weaker requirement than the condition of weak coupling to the side probes. (Indeed, for the quench shown in Fig. 2, $T_{l}$ and $T_{r}$ are each more than $30 \%$ of $T_{s}$.) The point is that for $\Delta \alpha<90^{\circ}$, trajectories cannot enter a side probe directly, since the injection or acceptance cones of two mutually perpendicular channels do not overlap. An electron approaching the side probe will be reflected (Fig. 3, left inset), and will then typically undergo multiple reflections in the junction region (right inset). This scrambles the trajectory and tends to equalize $T_{l}$ and $T_{r}$, thus reducing $R_{H}$. We find that scrambling is not very effective in the smooth hard-wall geometry considered, since an electron reflected from one side probe has a relatively large probability of entering the opposite side probe (this is the "rebound" mechanism for a negative $R_{H}$ of Ford et al. ${ }^{3}$ ).

The longitudinal resistance $R_{L} \equiv R_{25,16}$, shown in Fig. 4 , can also be discussed in terms of guiding and collimation. Guiding eliminates backscattering and hence drastically reduces $R_{L}$ for $B \gtrsim B_{g}$. The maxima of the oscillations on the Hall plateau (Fig. 1) correspond to complete guiding $\left(R_{H} \approx R_{0}\right)$, which is why they line up with the minima of the oscillations in $R_{L}$. Before the large decrease of $R_{L}$ there is a peak, leading to a "camel back" shape due to the destruction of collimation by a weak magnetic field (on the order of, but smaller than, $\left.B_{g}\right){ }^{20}$ To demonstrate this effect in a more direct way, we consider the bend resistance ${ }^{5} R_{B} \equiv R_{12,53}$, which involves the opposite current and voltage contacts 1 and 3 .

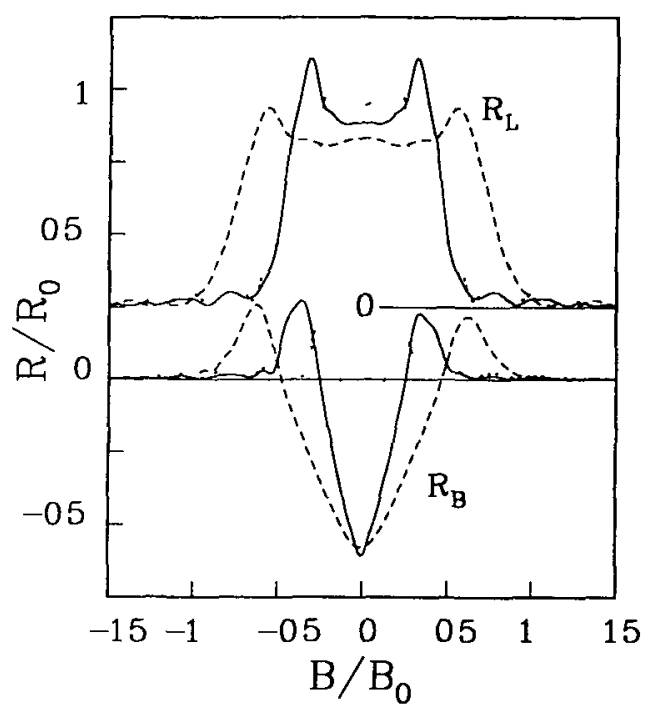

FIG. 4. Longitudinal resistance $R_{L} \equiv R_{25,16}$ and bend resistance $R_{B} \equiv R_{12,53}$ for the three hard-wall geometries of Fig. 1 ( $R_{L}$ has an offset of $0.25 R_{0}$ ). 